\title{
«Dall'altra parte della luna». Le poesie di Giorgio Bassani tra gli Stati Uniti e il Canada
}

"On the Other Side of the Moon". Giorgio Bassani's Poems Between the United States and Canada

"De l'autre côté de la lune ». Les poésies de Giorgio Bassani entre États-Unis et Canada

\section{Valerio Cappozzo}

\section{OpenEdition Journals}

Edizione digitale

URL: http://journals.openedition.org/cei/3925

DOI: 10.4000/cei.3925

ISSN: 2260-779X

Editore

UGA Éditions/Université Grenoble Alpes

\section{Edizione cartacea}

ISBN: 978-2-37747-040-2

ISSN: 1770-9571

Notizia bibliografica digitale

Valerio Cappozzo, ««Dall'altra parte della luna». Le poesie di Giorgio Bassani tra gli Stati Uniti e

il Canada», Cahiers d'études italiennes [Online], 26 | 2018, online dal 28 février 2018, consultato il 26 mars 2021. URL: http://journals.openedition.org/cei/3925 ; DOI: https://doi.org/10.4000/cei.3925

Questo documento è stato generato automaticamente il 26 mars 2021

(c) ELLUG 


\title{
«Dall'altra parte della luna». Le poesie di Giorgio Bassani tra gli Stati Uniti e il Canada
}

\author{
"On the Other Side of the Moon". Giorgio Bassani's Poems Between the United \\ States and Canada \\ "De l'autre côté de la lune ». Les poésies de Giorgio Bassani entre États-Unis et \\ Canada
}

Valerio Cappozzo

\begin{abstract}
«Ad ogni modo sia ben chiaro: niente di più dissimile della Milano di Hemingway da quella di Manzoni, dove, se la morte e il dolore corrono le strade, ogni donna ha però il viso di Lucia». Giorgio BASSANI ${ }^{1}$
\end{abstract}

Durante il corso di Letteratura italiana moderna e contemporanea tenutosi nel semestre primaverile del 2016 all'Università del Mississippi, abbiamo letto diverse poesie e passaggi narrativi di Giorgio Bassani. Il corso, dato il luogo geografico in cui si è svolto e seguendo l'interesse degli studenti, aveva lo scopo di soffermarsi su quella letteratura italiana che ha cercato, in un modo o nell'altro, rapporti e punti di contatto con la letteratura americana. Se «l'inizio della guerra 1914-1918 trovò i lettori di tutto il mondo a testa china sui romanzi russi», la seconda guerra mondiale spostò l'interesse verso quella statunitense, verso quei romanzi senza inquadramento storico, in parte da considerare "come i segni di una moda, anzi d'una infatuazione, più che come operazioni dell'intelligenza e del gusto» ${ }^{2}$. Alla luce di questo giudizio severo di Cecchi, abbiamo cercato di capire quale sia stata la necessità, durante il fascismo, di tradurre i romanzi americani o di farne, come nel caso di The Postman Always Rings Twice (1934), degli adattamenti cinematografici che potessero sfuggire alla censura. Se il naturalismo verghiano suscitava sospetti a causa delle allusioni al brigantaggio nella novella L'amante di Gramigna e il progetto di adattamento veniva così bocciato dal Ministero della Cultura Popolare, il diner descritto da James Cain era un luogo troppo 
indeterminato per destare sospetti e far pensare a storie più 'scomode' per il regime. Il gruppo di collaboratori alla rivista "Cinema», Alicata, De Santis e Puccini cominciarono così a lavorare alla riduzione cinematografica del romanzo poliziesco americano sotto la direzione di Luchino Visconti, che lo aveva letto in traduzione francese ai tempi in cui era stato assistente alla regia di Jean Renoir a Parigi ${ }^{3}$. Ossessione traduce, dalla California alla Bassa Padana, quell'idea del non-luogo dove è possibile ambientare una qualsiasi storia, sentendosi liberi dagli schemi sociali derivati da un eccesso di perbenismo e morale, ciò che appunto diversi scrittori e intellettuali italiani cercavano nella letteratura americana. In questa occasione, per andare alla ricerca delle locations dove girare alcune scene del film, viene chiamato un giovane scrittore esordiente, il ferrarese Giorgio Bassani, fine conoscitore dei luoghi che vanno dalla sua Ferrara a Codigoro, paesaggi cantati nelle sue poesie che proprio in quegli anni cominciavano a prendere forma. Usando un po' di immaginazione possiamo, effettivamente, calarci in una poesia di Storie dei poveri amanti, seguendo la telecamera guidata da Visconti che penetra nelle nebbie mattutine o attraversa i campi con la stessa indolenza del treno regionale Ferrara-Bologna, preso ogni giorno da Bassani per andare all'università, itinerario, come tutti gli spostamenti, fuori dallo spazio e dal tempo per la natura stessa del viaggio, il ricordo del quale rimane vivo nei versi di poesie quali, per esempio, Verso Ferrara ${ }^{4}$.

2 La connessione tra le ambientazioni di Ossessione, la letteratura americana e la sua poesia spingerà Bassani, due anni dopo l'uscita del film, a tradurre il romanzo di James Cain ${ }^{5}$. Il fatto che uno scrittore italiano traducesse in quegli anni la narrativa americana, e che nelle riviste di cui era redattore invogliasse alla traduzione e alla circolazione della letteratura statunitense, lo ha fatto sentire più vicino sia ai miei studenti di oggi, sia a quelli che ebbero la fortuna di averlo come professore quarant'anni fa all'Indiana University ${ }^{6}$. La forza di questi punti di contatto sono la ragione per cui durante il corso abbiamo letto alcuni passaggi del romanzo di Cain in lingua originale prima di vedere il film di Visconti per poi, finalmente, affrontare la lettura delle poesie di Bassani. In altre parole, abbiamo cercato di vedere come l'America sia stata adattata nell'Italia degli anni Quaranta, l'abbiamo guardata da lontano, pur standoci nel bel mezzo. Ciò è servito per capire che cosa gli intellettuali italiani degli anni Trenta e Quaranta hanno immaginato di quel continente così lontano e così sconfinato.

3 Lo sappiamo, «l'America è lontana / dall'altra parte della luna» ${ }^{7}$ e il suo mito ha caratterizzato la cultura del Novecento anche dopo il fascismo, negli anni Cinquanta con l'arrivo della Beat Generation, e più avanti con la televisione che ce la mostra continuamente nei format televisivi e nei telefilm. L'epoca di Bassani, digiuna dell'invasione dei mass media, leggeva l'America nei libri che sbarcavano in Italia, pronti per essere tradotti: per esempio, tra i primi, quelli di Herman Melville, Nathaniel Hawthorne, Henry James, Emily Dickinson, Truman Capote e John Steinbeck. Nello stesso momento si ragionava sul significato del nuovo mondo nei resoconti di viaggio scritti da quegli intellettuali che si avventuravano, per lunghi o brevi periodi, dall'altra parte dell'oceano, pagine piene di riflessioni sull'America e sull'aspirazione comune che andava a cercare in quell'altrove la propria realizzazione.

4 Mario Soldati, uno dei grandi amici di Bassani, ci visse dal 1929 al 1931 e nel suo taccuino di viaggio appunta, con grande lucidità e profondità, i diversi aspetti della propria esperienza. Pochi all'epoca erano gli intellettuali che affrontavano il viaggio 
americano: fra gli altri ricordiamo Luigi Barzini, corrispondente del «Corriere della Sera» dal 1921, e Giuseppe Prezzolini che insegnava a New York già dal $1923^{8}$. Soldati racconta il suo viaggio in forma diaristica e ci fa capire veramente cos'era quell'America ancora sconosciuta e simbolica. Nelle sue pagine intense si nota soprattutto l'italianità in rapporto a quell'estero su cui pretendiamo una qualche paternità per il fatto di averla scoperta con Cristoforo Colombo e contaminata con l'emigrazione di massa nel XX secolo. Camminando per le strade, così moderne $\mathrm{e}$ distanti dall'Europa, il ragionamento di Soldati affronta un aspetto cruciale del viaggio che diventa metaforico, e cioè si trasforma nel percorso della vita di ogni uomo che si è allontanato dal proprio luogo natio.

Il primo grande viaggio [...] crea quasi, nella mente, una nuova categoria: la categoria della lontananza; la considerazione, ormai, di tutte le terre lontane. È forse un vizio. Chi è stato in Cina vorrebbe provare l'Argentina, il Transvaal, l'Alaska. Chi è stato in Messico si commuove anche quando sente parlare dell'India, dell'Australia, della Cina. Questi nomi, una volta al più colorate e melanconiche geografie, sono ora possibili, reali, affascinanti. Chi ha provato la lontananza difficilmente ne perde il gusto. Il primo viaggio, la prima sera che il novo peregrin è in cammino, nasce la nostalgia, per sempre. Ed è il desiderio di tornare non soltanto in patria; ma dappertutto: dove si è stati e dove non si è stati. Due grandi direzioni si alternano; verso casa, verso fuori. L'ora volge il disio. Ma è, ormai, un desio. Un avvertirsi, comunque e sempre, lontani ${ }^{9}$.

Chi è in grado di raccontare per iscritto i viaggi, tanto da dare l'impressione al lettore di viaggiare lui stesso, è uno scrittore abile e apprezzato. L'idea del viaggio si sposa perfettamente, sin dall'alba dei tempi omerici, con la letteratura e con la forma del racconto perché ogni passaggio, ogni avvenimento sorprendente e inatteso che solo in viaggio può accadere, corrisponde a un momento nella formazione della persona che crea la propria esperienza giorno dopo giorno, modellandosi a seconda degli eventi che la vita le offre. Rendere vicine le cose lontane e viceversa, è la magia dello scrivere, come è l'esperienza del viaggiatore che attraversa diversi continenti ${ }^{10}$.

6 A Mario Soldati, non a caso, è dedicata la poesia Campus scritta in Indiana e pubblicata in In gran segreto, poesia sul rapporto di Bassani con gli studenti americani. Infatti, la sua esperienza professorale all'Indiana University nel 1976 e il suo ritorno nel '77, ha lasciato un segno che si è poi concretizzato nelle traduzioni di alcune sue poesie e in saggi critici scritti da chi lo ha conosciuto come professore o come collega. Addirittura quarant'anni dopo ne stiamo ancora celebrando il viaggio con l'uscita del libro Lezioni americane di Giorgio Bassani ${ }^{11}$, e con le tante presentazioni che sono state fatte e che si faranno in giro per il mondo, a dimostrazione dell'interesse per lo scrittore ferrarese e la sua biografia, oltre che, naturalmente, per l'opera narrativa e in versi. L'affetto, perché proprio di questo sentimento credo si tratti, dei lettori americani, russi, francesi, tedeschi, argentini, coreani, è un dato notevole e fa sì che i suoi scritti continuino a essere tradotti e letti in tanti paesi ${ }^{12}$.

7 Siamo ritornati al punto di partenza, a quei «confronti infra ed extra» ${ }^{13}$ che ci hanno portato in una classe di letteratura nel Mississippi, in un diner californiano e fatto viaggiare in treno attraverso i paesaggi della Bassa Padana alla ricerca di una location dove poter raccontare una storia, più personale che letteraria, per Soldati come per Bassani. Ma l'America è anche amara, continente scoperto grazie a un errore nel calcolo della navigazione, e diventato subito terra di oppressione cruenta contro i nativi e, nei secoli più recenti, sede del capitalismo sfrenato che condiziona le economie occidentali, 
pur rimanendo la meta del viaggio per eccellenza, il territorio dell'adattabilità di ognuno e di ogni cosa.

È molto bello, forse la cosa superiormente bella in una traversata, starsene ore e ore a guardare lungo il fianco della nave i giuochi dell'acqua, i lineamenti del mare. Guardarli fino alla stanchezza delle palpebre e delle pupille. Finché in quella misteriosa e suntuosa uniformità, in quell'algebra smaltata e sfavillante, si palesa e si sviluppa un disegno, un ritmo, un movimento. [...] Lo sgranarsi dell'onde, i festoni e le gale delle tinte, le bianche medaglie della spuma, formano una sorta di decorazione, nella quale l'energia della natura sembra esprimersi come in una musica visiva, che continuamente rinasce dalla sua decomposizione continua. Non senza diretto rapporto, è qualcosa come l'arte dei popoli delle solitudini marine e delle solitudini desertiche. Come l'ornamentazione geometrica sui vasi della prisca grecità. E come l'arte decorativa degli arabi e moreschi; non meno che degli indoamericani ${ }^{14}$.

8 Con l'idea del viaggio in mente, secondo la quale, tornando a Soldati, partire è incominciare un percorso di lontananza in cui, parafrasando Cecchi, l'onda franta dal piroscafo in navigazione atlantica comincia a essere tutte le acque del mondo, tradurre la letteratura americana corrispondeva evidentemente a un'esigenza, al viaggiare pur stando fermi e lasciare che la narrativa diventasse internazionale, superasse cioè la costrizione della lingua. Elio Vittorini e Cesare Pavese sono traduttori autodidatti che non sono mai stati in America e che hanno imparato l'inglese in Italia, commettendo errori nelle traduzioni che facevano per seguire una passione e un bisogno impellente. «Per tradurre bene, bisogna innamorarsi della materia verbale di un'opera, e sentirsela rinascere nella propria lingua con l'urgenza di una seconda creazione» ${ }^{15}$. L'esigenza di una seconda chance era scaturita dalla speranza di rimediare agli errori commessi, affinché l'«'io' lirico, protagonista della creazione» si ridestasse e prendesse coscienza di sé ${ }^{16}$. Cesare Pavese cominciò a tradurre i capolavori della letteratura americana spinto dalla voglia di esplorare con il pensiero una terra primordiale che si offriva a tutti, un mondo nuovo insieme fecondo e barbarico, appesantito dal passato europeo ma ancora a suo modo innocente. E infatti non si limita a tradurre i romanzi, ma nei suoi saggi discute le ragioni e la necessità dell'importare nell'Italia fascista questa narrativa d'oltreoceano, accorgendosi bene che l'America attirava per il fatto di essere un paese estero, un ideale inizio della storia, ma anche «il gigantesco teatro dove con maggiore franchezza che altrove veniva recitato il dramma di tutti [...]. La cultura americana ci permise in quegli anni di vedere svolgersi come su uno schermo gigante il nostro stesso dramma» ${ }^{17}$. La teatralità, in questo caso e, secondo Pavese, a ragion veduta, significava la versatilità con la quale il territorio statunitense proponeva un fondale alla libera iniziativa di ognuno, e questo fu il motivo per cui si scelse il romanzo di James Cain per la sceneggiatura e l'ambientazione di Ossessione. Terra riscoperta nel momento giusto e anche al posto giusto, lontana cioè dal grigiore del primo dopoguerra e dalla tragedia della seconda guerra mondiale. Con lo stesso intento Fernanda Pivano traduce i poeti della Beat Generation, sapendo che all'entrata in crisi del linguaggio, delle istituzioni e della società in generale, quei versi americani insegnavano un diverso modo di vivere, attraverso un modo nuovo di esprimersi ${ }^{18}$. Anni più tardi, dopo le lezioni di Bassani all'Indiana University, Italo Calvino, che non terrà le sue lezioni in America, nei suoi appunti sottolinea la leggerezza, la rapidità, l'esattezza, la visibilità e la molteplicità di forme letterarie che liberano i contenuti e la capacità di esprimersi senza quel peso che tanto aveva attanagliato gli scrittori italiani ed europei testimoni delle due guerre mondiali. Questa era la 'nuova' America a cavallo degli anni Settanta e 
Ottanta, la stessa in cui Bassani aveva viaggiato e lasciato un'impronta con il suo insegnamento e con le sue poesie di cui In gran segreto, che ne contiene alcune scritte proprio negli Stati Uniti, rimane testimonianza ${ }^{19}$.

Recentemente ho avuto il piacere di parlare di questi argomenti con Nancy D'Antuono, professoressa d'Italiano, ora in pensione, al Saint Mary's College a Notre Dame in Indiana che, all'epoca della permanenza di Bassani, si recava spesso a Bloomington.

Ho avuto l'occasione di incontrare Bassani più volte, sempre grazie alla generosità del Professor Lèbano che spesso organizzava degli incontri tra gli studenti di Italianistica a cui invitava anche la gente, come me, che parlava italiano. Durante quelle occasioni mi è capitato di discorrere a lungo con Bassani sulle differenze tra l'Italia e gli Stati Uniti, e io credo che dell'America lui apprezzasse soprattutto l'apertura geografica e intellettuale pronta alle nuove idee, proprio perché è un paese giovane in cui la letteratura e la creatività dipendono dalla giovinezza della sua storia che conferisce loro una certa leggerezza che sicuramente ha aiutato Bassani a sentirsi diverso e a riscoprire la sua scrittura sotto una nuova luce ${ }^{20}$.

In America Bassani viene letto sin dal 1960 con la pubblicazione di The Gold-rimmed Spectacles che, insieme a The Garden of the Finzi-Continis nel 1965, esce per le edizioni anglo-americane di Atheneum Books nella traduzione di Isabel Quigly ${ }^{21}$. Dalle edizioni successive il traduttore diventa William Weaver e L'Airone, Il romanzo di Ferrara e Dietro la porta escono in America nel 1970 e nel 1972, L'odore del fieno nel 1975 e la riedizione de Il giardino dei Finzi-Contini, nella nuova traduzione, nel $1977^{22}$. Il successo dello scrittore ferrarese si rinforza per la vincita del premio Oscar da parte del film di Vittorio De Sica, anche se, come noto, la sceneggiatura è stata rifiutata dall'autore. Il suo interesse per la letteratura americana, che si era concretizzato sin dagli anni Cinquanta con la pubblicazione di romanzi americani in traduzione sulle riviste «Botteghe oscure»e «Paragone», ora viene ricambiato.

11 Ma che cosa, della scrittura di Bassani, ci sembra legittimo chiedere, viene apprezzato in America? Alla poesia gli studenti americani preferiscono tendenzialmente la narrativa, genere al quale la loro stessa letteratura è più avvezza. Qui a Oxford nel Mississippi, casa di William Faulkner (1897-1962) e ancor oggi punto d'incontro di diversi scrittori del sud, la narrativa, come la musica blues, gospel e jazz, è usata come strumento per raccontare i conflitti razziali che hanno caratterizzato la storia di questo paese dal XVII secolo in poi. Ma la poesia e la narrativa di Giorgio Bassani trovano lettori attenti e interessati che nelle sue pagine leggono la denuncia del fascismo e della persecuzione degli esseri umani, relazionandola alla storia degli afro-americani. Così anche nei writers del sud si trova il bisogno costante di essere testimoni, di incarnare la memoria, di riaffermare la storia del proprio tempo attraverso l'atto stesso della scrittura. Ebraismo o negritudine, Italia o America, Nord o Sud, si tratta sempre di una ricerca di libertà dall'oppressione di un gruppo di uomini su altri gruppi. Questi sono in larga misura i temi di cui ho discusso con i miei studenti americani per avvicinare uno scrittore italiano a chi, pur avendo scelto di imparare la nostra lingua e parte della nostra cultura, ha bisogno di trovare delle assonanze. Nella terra sudista raccontata da tanti, tra cui emergono Mark Twain (1835-1910), Ross Barnet (1898-1987), Richard Wright (1908-1960), Eudora Welty (1909-2001), Tennessee Williams (1911-1983), Elmore Leonard (1925-2013) e Willie Morris (1934-1999), Giorgio Bassani ha suscitato l'interesse degli studenti proprio con la sua narrativa di denuncia. Per questo motivo abbiamo letto le sue pagine e ci siamo soffermati sulle poesie sia in italiano sia in traduzione inglese prima e a ridosso delle sue visite nel Midwest, dove ha insegnato e dove ha 
scritto i versi di Campus e di Visitando l'Indiana, oltre a rielaborare quelli composti in giro per la California, l'Illinois e il Michigan.

Le prime traduzioni in inglese delle sue poesie risalgono addirittura al 1950 e vengono pubblicate negli Stati Uniti nel volume curato da Marguerite Caetani, An Anthology of New Italian Writers, dove ne compaiono otto, riprese da Te lucis ante, e tradotte, come sarà per i romanzi, da William Weaver ${ }^{23}$. Queste traduzioni introducono in Nord America Bassani come poeta ancor prima che venga conosciuto come narratore. È interessante, infatti, notare che la sua nota biografica, a conclusione del volume, che include anche il racconto breve Una storia d'amore, apparso la prima volta con il titolo Storia di Debora in Una città di pianura nel 1940, lo presenta esclusivamente come poeta ${ }^{24}$. Questo è un punto che ci farà capire meglio le sue dichiarazioni sulla prosa dipendente dalla poesia, come lui stesso era solito affermare ${ }^{25}$.

Nel 1982 viene pubblicato a Toronto l'unico libro di poesie di Bassani in traduzione inglese, Rolls Royce and Other Poems, che ne contiene dodici ${ }^{26}$, tradotte da Francesca Valente in collaborazione con Greg Gatenby e Irving Layton, sotto la supervisione di Bassani stesso e di Portia Prebys. Nell'introduzione alla silloge, il grande critico canadese Northrop Frye sottolinea che le poesie di Bassani assomigliano a i tentativi che facciamo quando cerchiamo di ricordare degli aspetti della nostra identità che, appena sfiorati, ci sfuggono. La stessa cosa succede per i nomi che non si ricordano ma che si è sicuri di poter ricordare e, finché ci si pensa, e anzi più ci si pensa, meno tornano alla mente, come se fossero dimenticati per sempre. Ma a volte accade l'esatto opposto, la memoria cioè riaffiora improvvisamente e, come per Proust con le memorie involontarie, il fatto sembra voler indicare, che siamo stati noi ad allontanarcene.

If we try to recall our identity from conscious memories of the past, it retreats like a forgotten name, until we give up trying. But sometimes, when our minds are on something else, the movement is reversed: the memory comes out, as it did to Proust, touches the rememberer, and says, in several senses, "here you are" 27 .

Con il Canada Bassani ha avuto una frequentazione intensa grazie a Stelio Cro, professore della McMaster University nella città di Hamilton in Ontario. Lì Bassani tenne delle conferenze e insegnò nel semestre primaverile del 1978, due anni dopo essere stato Visiting Professor all'Indiana University, e tornerà in Canada per insegnare alla Queens University, a Kingston in Ontario, l'anno successivo. In Canada tenne un corso sulla propria narrativa e un secondo sull'opera narrativa di Moravia, Cassola e Pasolini. Con l'occasione della sua visita, Stelio Cro riuscì a inaugurare la rivista «Canadian Journal of Italian Studies», rimasta attiva dal 1977 al 1997. Scrive Cro: "Questo numero è dedicato a Giorgio Bassani, l'illustre scrittore ferrarese che tra breve ci visiterà. L'idea della fondazione di questa rivista è maturata da questa fortunata circostanza. Egli ha dato alla rivista alcuni poemi inediti affinché se ne fregiasse il primo numero $»^{28}$. Anche il secondo numero della rivista è dedicato allo scrittore, come indica il titolo, Omaggio a Giorgio Bassani (II), e contiene tra i vari articoli otto poesie che sarebbero apparse la primavera successiva nell'edizione Mondadori intitolata, in un primo momento Dittico, e poi In gran segreto:

The Canadian Journal of Italian Studies is pleased to include eight more inedited poems by Giorgio Bassani in the Winter issue. They are part of his last book of poetry entitled In gran segreto (formerly Dittico) taken from the last of these "American Poems," as Bassani refers to them, inspired by his visit to the United States in 1976 where he was Visiting Professor of Italian at Indiana University (Bloomington) ${ }^{29}$. 
Un'altra traduzione delle poesie di Bassani verrà fatta negli Stati Uniti nel 1984 da Mark Musa $^{30}$. Professore di letteratura italiana e collega di Edoardo Lèbano all'Indiana University, conobbe bene Bassani durante le sue due visite nell'università statunitense e durante la seconda lo ospitò a casa sua. Avevano avuto occasione di parlare spesso anche fuori dall'università, soprattutto a casa di Lèbano, dove si organizzavano cene con gli altri professori e con gli studenti di dottorato che seguivano le lezioni con lo scrittore. Durante l'ultima cena, prima di ripartire per Roma, Bassani regalò agli amici più stretti una poesia trascritta di suo pugno pubblicata in Epitaffio, Davvero cari non saprei dirvelo. Dopo esser stata tradotta ed edita nel libro canadese a cui abbiamo già fatto riferimento, Musa stesso la tradusse qualche anno dopo con varianti significative, come si constata leggendo in parallelo le due traduzioni, qui precedute dal testo italiano della poesia:

\begin{tabular}{|c|c|}
\hline \multicolumn{2}{|c|}{$\begin{array}{l}\text { Davvero cari non saprei dirvelo } \\
\text { Davvero cari non saprei dirvelo } \\
\text { attraverso quali } \\
\text { strade così di lontano } \\
\text { io sia riuscito dopo talmente } \\
\text { tanto tempo a tornare }\end{array}$} \\
\hline $\begin{array}{l}\text { I Really Couldn't Say, Friends } \\
\text { I really couldn't say, friends, } \\
\text { along which road } \\
\text { from so far away } \\
\text { I managed after such a long time } \\
\text { to find my way back. } \\
\text { I can only tell you that I let } \\
\text { myself be led in darkness } \\
\text { by someone who took } \\
\text { me, silently, by the } \\
\text { hand }{ }^{32}\end{array}$ & $\begin{array}{c}\text { Believe Me, My Friends, } \\
\text { I Can't Begin to Tell You } \\
\text { Believe me, my friends, I can't begin to tell you how } \\
\text { by what means or } \\
\text { what roads so far away } \\
\text { I happened to succeed after so long a stretch } \\
\text { of time to make it back again }\end{array}$ \\
\hline
\end{tabular}

16 Anche se la traduzione a cura di Francesca Valente fu supervisionata dallo stesso Bassani, quella di Musa sembra più attenta al profondo significato delle parole che all'aspetto formale del componimento. Partendo dal titolo, è evidente che tra «I really couldn't say, friends» e «Believe me my friends, I can't begin to tell you how», la differenza è considerevole non solo nella lunghezza del verso, ma nell'intensità 
dell'espressione. Se nel primo caso il «davvero» è tradotto con un preciso ed esclamativo «really», nel secondo viene colto il tono con «believe me», una sorta di «credetemi perché mi è veramente difficile dirvi quanto...». Anche se la prima traduzione mantiene il condizionale «I couldn't», come nell'originale «non potrei», non eguaglia la forza della condizione che sta, anche in italiano, non solo nel tempo verbale, ma nella confessione della difficoltà di esprimersi per essere appieno compreso. Anche la versificazione originale viene rispettata nella traduzione di Musa, ma non in quella di Valente, e sappiamo quanto siano importanti, in Epitaffio, la metrica, l'interpunzione e la disposizione delle parole al centro della pagina, come, successivamente, nelle poesie di In gran segreto. L'aver seguito le lezioni di Bassani sulla propria opera in versi e l'aver ricevuto la poesia in dono, durante l'ultima serata americana dello scrittore, ha influito sulle scelte e sulla qualità della traduzione.

Durante il corso di Letteratura italiana moderna e contemporanea, tenutosi nel semestre primaverile del 2016 all'Università del Mississippi, abbiamo letto le traduzioni delle poesie di Bassani sottolineando le differenze, che sono state utili per tornare alle versioni originali e capirne meglio l'intenzione, l'ispirazione, il lessico. Abbiamo anche avuto un'occasione particolare, l'incontro con il poeta afro-americano Cyrus Cassells, che ci ha aiutato a cogliere questioni di cui abbiamo fatto cenno anche in queste pagine, come il capire l'opera di un italiano ebreo confrontandola con quella degli scrittori del sud degli Stati Uniti e avvertirne meglio la condizione storica e privata affidata alla scrittura poetica. Nato nel 1957, Cassells insegna scrittura creativa alla Texas State University a San Marcos in Texas. Ha vinto diversi premi letterari ed è stato candidato al Premio Pulitzer per la poesia nel $1994^{34}$. Ama molto l'Italia e la letteratura italiana e, nell'autunno del 1996, cenò a Roma con Giorgio Bassani, episodio di cui ci ha reso partecipi.

Una sera cenammo Da Luigi, la sua trattoria preferita a Piazza Sforza Cesarini. È un ristorante tipico romano aperto dal 1960 tra Corso Vittorio Emanuele III e Via dei Banchi Vecchi. La cena fu rilassata e informale ma subito mi cominciò a raccontare, visto che ovviamente io non ero di Roma, di quando lui ci arrivò clandestinamente nel '44. Rimasi molto sorpreso che un uomo come lui avesse avuto quel passato anche se, certo, in quel momento storico il rischio di deportazione per un ebreo era altissimo. Fui colpito nel sentirlo parlare di quegli argomenti e non facevo altro che pensare alla sua eleganza e d'un tratto a immaginarmi le sue fughe dalla follia nazista. L'una e l'altra cosa mi sembravano così distanti... Qualche anno fa poi sono tornato a Ferrara per un vero e proprio pellegrinaggio letterario e sono stato al cimitero ebraico, ho camminato per le vie del centro, l'ho percorsa più volte in lungo e in largo e respirandone l'aria, ho scritto questa poesia ${ }^{35}$ generata, direi, da un riflesso spontaneo: 


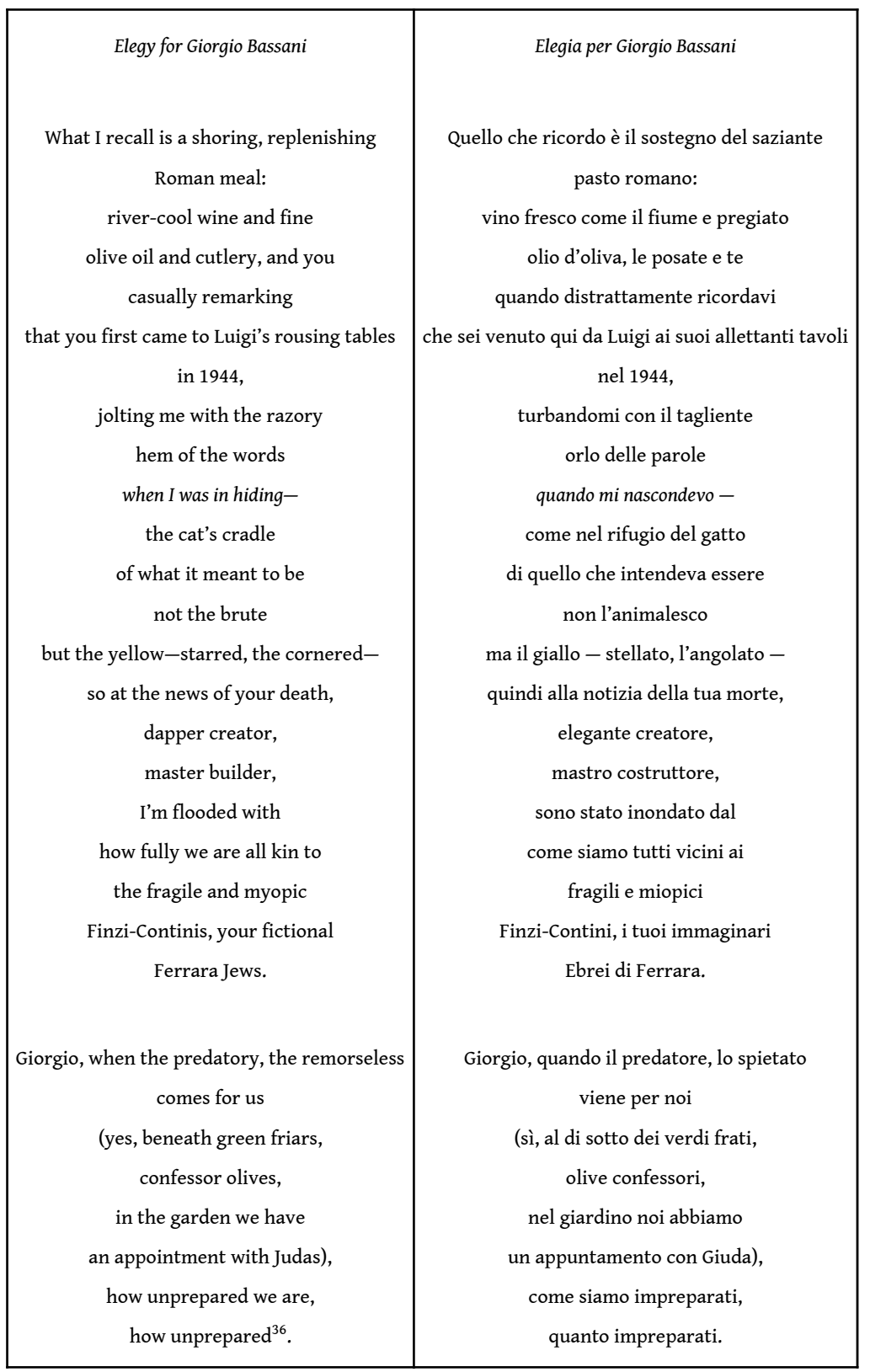

Il corso di Letteratura si è concluso con una testimonianza che agli studenti è servita per capire quanto il tempo e le tracce che gli uomini vi lasciano impresse siano esempi di cammini che si possono ripercorrere. Nel semestre primaverile del 2016, rileggendo le poesie di Giorgio Bassani, i suoi romanzi e la letteratura statunitense del sud, ci è sembrato di avere intrapreso un viaggio a un tempo reale e simbolico - come quello dei personaggi di Manzoni e di Hemingway citati in esergo - viaggio nel corso del quale siamo venuti a trovarci «a metà strada tra Ferrara e la luna» ${ }^{37}$. 


\section{NOTE}

1. G. Bassani, I bastioni di Milano, in Id., Opere, a cura di R. Cotroneo, Milano, Mondadori, 1998, p. 1020. Il saggio fu pubblicato per la prima volta, come recensione a A Farewell to Arms (1929), sotto il titolo Ernest Hemingway milanese, su «Il Mondo», 3 agosto 1946, pp. 4-5. Bassani aveva tradotto il romanzo nel 1943, ma la sua traduzione non fu pubblicata e andò poi perduta. In Italia la pubblicazione del romanzo fu vietata dalla censura fascista fino al 1945, quando fu edito con il titolo Un addio alle armi nella traduzione di Bruno Fonzi per la Jandi Sapi di Milano, e di nuovo l'anno successivo da Mondadori nella traduzione di Fernanda Pivano e con otto illustrazioni di Renato Guttuso.

2. E. Cecchi, Introduzione, in E. Vittorini (a cura di), Americana, Milano, Bompiani, 1942, p. IX.

3. Anche Moravia partecipò alla revisione finale della sceneggiatura ma sotto falso nome a causa delle leggi razziali. Sulla censura subita da questo film, si veda: L. Miccichè, Visconti e il neorealismo: Ossessione, La terra trema, Bellissima, Venezia, Marsilio, 1998.

4. "Questa è l'ora che vanno per calde erbe infinite / nel mio paese gli ultimi treni, con fischi lenti / salutano la sera, affondano indolenti / in sonni dove tramontano rosse città turrite. // Dai finestrini aperti il vino delle marcite / monta al madido specchio delle povere panche; / dei giovanili amanti scioglie le dita stanche, / fa deserte di baci le labbra inaridite» (G. Bassani, Storie dei poveri amanti, Roma, Astrolabio, 1946, p. 26). Sulla poesia di Bassani si vedano: A. Dolfi, Le forme del sentimento: prosa e poesia in Giorgio Bassani, Padova, Liviana Editrice, 1981; Ead., Giorgio Bassani: una scrittura della malinconia, Roma, Bulzoni, 2003; Ead., Dopo la morte dell'io. Percorsi bassaniani «di là dal cure», Firenze, Firenze University Press, 2017; G. Varanini, Bassani, narratore, poeta, saggista, Modena, Mucchi, 1991; M. Gialdroni, Giorgio Bassani, poeta di se stesso. Un commento al testo di «Epitaffio» (1974), Berna, Peter Lang, 1996; A. Guiati, L'invenzione poetica. Ferrara e l'opera di Giorgio Bassani, Fossombrone, Metauro Edizioni, 2001; P. Pieri, Poesia e verità in Giorgio Bassani, in A. Perli (a cura di), Giorgio Bassani: la poesia del romanzo, il romanzo del poeta, Ravenna, Giorgio Pozzi Editore, 2011, pp. 17-31; Id., Un poeta è sempre in esilio. Studi su Bassani, Ravenna, Giorgio Pozzi Editore, 2012; M. Rueff, «Alas poor Emily». Bassani poeta, in R. Antognini e R. Diaconescu Blumenfeld (a cura di), Poscritto a Giorgio Bassani. Saggi in memoria del decimo anniversario della morte, Milano, LED, 2012, pp. 387-426; A. Luzi, Esperienza vissuta e scrittura nella poesia di Bassani, «Ermeneutica letteraria», $\mathrm{n}^{\circ}$ 9, 2013, pp. 65-82; F. Santi, Bassani poeta, ovvero Bassani e basta. Sonatina in cinque movimenti, «Nuovi Argomenti», $\mathrm{n}^{\circ}$ 72, ottobre-dicembre 2015, a cura di F. Longo, pp. 77-92; V. Cappozzo, Un attimo prima di cominciare a leggere. Dall'Ermetismo alle poesie di Giorgio Bassani, in A. Perli (a cura di), Il tempo dello spirito. Saggi per il centenario della nascita di Giorgio Bassani, «Sinestesie. Rivista di studi sulle letterature e le arti europee», vol. XIV, 2016, pp. 45-60; F. Bartolini, Le prime raccolte poetiche di Bassani tra naturalismo elegiaco e impegno civile, in S. Amrani e M. P. De Paulis-Dalembert (a cura di), Bassani nel suo secolo, Ravenna, Giorgio Pozzi Editore, 2017, pp. 161-197.

5. Su questa traduzione dall'inglese, la seconda alla quale Bassani si accinse dopo quella di Addio alle armi di Hemingway, si veda: R. Antognini, Giorgio Bassani e James Cain. Storia e critica di una traduzione, in V. Cappozzo (a cura di), Lezioni americane di Giorgio Bassani, Ravenna, Giorgio Pozzi Editore, 2016, pp. 89-121.

6. Sull'esperienza all'Indiana University mi permetto di rimandare al mio: Il viaggio in America di Giorgio Bassani tra poesia e insegnamento, in Lezioni americane di Giorgio Bassani, cit., pp. 15-39.

7. L. Dalla, Anna e Marco, dall'album Lucio Dalla, RCA Italiana, 1979.

8. Di Barzini si vedano: Gli americani sono soli al mondo, Milano, Mondadori, 1952 e O America: When You and I Were Young, New York, Harper \& Row, 1977 (traduzione italiana: O America! Eravamo giovani assieme, Milano, Mondadori, 1978). Di Prezzolini si veda America in pantofole: un impero senza imperialisti. Ragguagli intorno alla trasformazione degli Stati Uniti dopo le guerre mondiali, Firenze, 
Vallecchi, 1950; ripubblicato, con prefazione di Sergio Romano nel 2002 per la stessa casa editrice fiorentina.

9. M. Soldati, America primo amore, Firenze, Bemporad, 1935, pp. 14-15. Le citazioni in corsivo sono rimandi danteschi all'VIII canto del Purgatorio, citazioni più che pertinenti se si pensa che la montagna del Purgatorio è situata, secondo il poema sacro, al di là delle Colonne d'Ercole, dunque nell'oceano Atlantico in direzione dell'America. Questi i primi sei versi del canto: «Era già l'ora che volge il disio / ai navicanti e 'ntenerisce il core / lo dì c'han detto ai dolci amici addio; / e che lo novo peregrin d'amore / punge, se ode squilla di lontano / che paia il giorno pianger che si more» (corsivi miei).

10. È Soldati stesso a confessarci la sua impressione: «Durante il mio primo soggiorno americano, credevo che fosse possibile evadere: cambiare di patria, di religione, di ricordi, e di rimorsi. E vissi più di un anno nella morbosa persuasione di esserci riuscito. Il primo amore e il primo viaggio son malattie che si somigliano» (ivi, p. 87).

11. In Lezioni americane di Giorgio Bassani sono raccolti quattro saggi critici, oltre alla Premessa di Paola Bassani che ricostruisce le tappe critiche che hanno portato al libro e il ricordo del professor Edoardo Lèbano, che invitò Bassani all'Indiana University come Visiting Professor of Italian nel semestre primaverile del 1976. Il sottoscritto si è occupato del viaggio americano di Bassani e dell'esperienza di insegnamento della propria opera in prosa e in rima; Alessandro Giardino delle influenze della narrativa di Melville e Hawthorne su quella di Bassani; su quelle di Henry James ha lavorato Sergio Parussa e Roberta Antognini sulla traduzione di Bassani di The Postman Always Rings Twice. Il volume è inoltre corredato di un'appendice ricca di documenti: l'intervista di Stelio Cro a Bassani nel 1977; un articolo sulle poesie di Epitaffio scritto da Linda Nemerow-Ulman, che è stata studentessa di Bassani all'Indiana University; le lettere autografe e dattiloscritte di Bassani al professor Lèbano e infine la stesura originale della poesia Campus scritta appunto in Indiana nel 1976. Questo è l'indice: Paola Bassani, Premessa (pp. 9-10); Edoardo Lèbano, Giorgio Bassani a Indiana University (pp.11-13); Valerio Cappozzo, Il viaggio in America di Giorgio Bassani tra poesia e insegnamento (pp. 15-39); Alessandro Giardino, Ellissi eccentriche: Giorgio Bassani, Hermann Melville e Nathaniel Hawthorne (pp. 41-66); Sergio Parussa, L'odore della poesia. Giorgio Bassani e Henry James (pp. 67-87); Roberta Antognini, Giorgio Bassani e James Cain. Storia e critica di una traduzione (pp. 89-121). Appendici: Stelio Cro, Intervista a Giorgio Bassani (1977, pp. 125-134); Linda Nemerow Ulman, Visual Memory and the Nature of Epitaph: Bassani's "Epitaffio" (1986, pp. 135-148); Lettere di Giorgio Bassani a Edoardo Lèbano (1975-1977, pp. 149-160); Giorgio Bassani, Dal Campus (marzo 1976, pp. 161-165).

12. Qui si sta facendo riferimento alle diverse presentazioni del libro Lezioni americane di Giorgio Bassani e ai diversi interventi di Paola Bassani e del sottoscritto relativi all'esperienza americana dello scrittore ferrarese in occasione del centenario della nascita durante il quale, più che in altri momenti, ci sono state occasioni di incontro con diversi, e tra loro diversissimi, pubblici. Le presentazioni del libro si sono svolte all'Istituto Italiano di Cultura di New York, Washington D.C., a Francoforte (nella sede del Consolato d'Italia, ufficio culturale), e Mosca; nell'università dell'Indiana e in quella di Ferrara. Paola Bassani è stata invece a Seul, a Buenos Aires, a Toronto, a Berlino, a Bruxelles, a Londra, a Lisbona, a Barcellona e a Parigi.

13. G. Bassani, Campus, in Id., In gran segreto, Milano, Mondadori, 1978, p. 62.

14. E. Cecchi, America amara, Firenze, Sansoni, 1943 (1940), pp. 399-400. Emilio Cecchi fece due lunghi viaggi negli Stati Uniti e in Messico, nel 1930-1931 e nel 1937-1938.

15. C. Pavese, Lettere, a cura di L. Mondo e I. Calvino, vol. I, Torino, Einaudi, 1966, p. 554, lettera alla casa editrice Bompiani del 15 gennaio 1940. La stessa citazione si trova in esergo all'articolo sulla traduzione di Roberta Antognini, Giorgio Bassani e James Cain. Storia e critica di una traduzione, cit., p. 89. Vittorini cominciò a imparare l'inglese da un amico fiorentino, «poi continuai da solo, un po' come un sordomuto, su testi ancora di De Foe, e su autori del Settecento, su autori dell'Ottocento, su autori contemporanei anche americani fino al giorno in cui mi trovai in grado 
di poter tradurre correttamente» (E. Vittorini, Della mia vita fino a oggi raccontata ai miei lettori stranieri, in Id., Pesci rossi, marzo 1949). Per un approfondimento sulla traduzione nel Novecento si veda il ricco volume miscellaneo: A. Dolfi (a cura di), Traduzione e poesia nell'Europa del Novecento, Roma, Bulzoni Editore, 2004.

16. E. Vittorini, Diario in pubblico, Milano, Bompiani, 1970, p. 166. Elio Vittorini vede bene gli estremi di questa terra selvaggia che se da una parte è un luogo leggendario, dall'altro è quello dove si emigra per ricrearsi: «Sembra che i Padri Pellegrini fossero venuti dall'Europa pieni di delusioni e di stanchezza: per finire, non per cominciare. [...] Trovarono in America la necessaria ferocia per praticare quei pregiudizi feroci; essere in qualche modo vivi» (ivi, p. 116).

17. C. Pavese, La letteratura americana e altri saggi, Milano, Il Saggiatore, 1959, pp. 195-196. Oltre alle traduzioni e agli studi di Vittorini e di Pavese, è importante tenere presenti anche altri testi in cui si leggono le traduzioni di autori americani o si affrontano le prime analisi critiche dedicate alla letteratura americana come, per esempio: G. Baldini (a cura di), Poeti americani (1662-1945), Torino, Francesco De Silva, 1949; L. Anceschi, Poetica americana e altri studi contemporanei di poetica, Pisa, Nistri-Lischi Editori, 1953.

18. Sulle ragioni della traduzione, si veda: F. Pivano, Vittorini traduttore e la cultura Americana, dibattito con la partecipazione di Claudio Gorlier, Carlo Izzo, Agostino Lombardo, Fernanda Pivano, in Omaggio a Elio Vittorini, Terzo programma 3, 1966, pp. 152-161.

19. Le poesie Campus e Visitando l'Indiana sono pubblicate in In gran segreto, cit., pp. 64, 70-71.

20. Nancy L. D'Antuono si è occupata nella sua carriera di diversi aspetti della letteratura italiana, comparata a quella spagnola, si veda per esempio: N. D'Antuono, Boccaccio's «Novelle» in the Theater of Lope de Vega, Madrid, Porrúa Turanzas, 1983.

21. Di Isabel Quigly è la traduzione di A prospect of Ferrara, edito a Londra da Faber and Faber nel 1962.

22. Tutti i romanzi di Bassani sono usciti nella sua traduzione a New York per la casa editrice Harcourt: The Heron, 1970; Five Stories of Ferrara, 1971; Behind the Door, 1972; The Smell of Hay, 1975; The Garden of the Finzi-Continis, 1977, riedito nel 2005 sempre a New York da Everyman's library.

23. An Anthology of New Italian Writers. Edited by Marguerite Caetani and selected from the pages of the review Botteghe Oscure, Westport, Greenwood Press, 1970 (1950), pp.91-94. Le poesie tradotte sono nell'ordine: Valle dell'Aniene, Dal carcere, Vide cor meum, Per un quadro di Morandi, Ars poetica, L'alba ai vetri e In Memoriam (che contiene le poesie Sogno e A mio padre). Questa antologia comprende i seguenti autori: Mario Soldati (The Window), Giorgio Bassani (Poems and Love Story), Tommaso Landolfi (Cancroregina), Attilio Bertolucci (selezione da The Indian Hut), Giuseppe Dessì (Angel Island), Giorgio Caproni (The Funicular), Joyce Lussu (due racconti, The Matriarch e The Bambina), Franco Fortini (Poems), Vasco Pratolini (The Girls of Sanfredino), Alfonso Gatto (Novel 1917), Antonio Rinaldi (Poems); Roberto Roversi (Poems for a Print-Collector), Guglielmo Petroni (The House is Moving).

24. «Giorgio Bassani, though born in Bologna March 4, 1916, has always lived in Ferrara till 1943, and now lives in Rome. He has published: Storie dei poveri amanti (1946), Te lucis ante (1948), Storia d'amore and a few essays. He won one of the Premi Roma for 1950 with a small volume of poetry which will soon be published by Mondadori [Un'altra libertà, 1951]. He is associate-editor of Botteghe Oscure» (ivi, p. 475).

25. Celebri le seguenti affermazioni: «Quanto a me, io non sono un romanziere, o un rimatore, o un saggista. Sono un poeta, [...] sostanzialmente un poeta» (Un'intervista inedita (1991), in G. Bassani, Opere, cit., p. 1347); «Io sono abbastanza conosciuto come romanziere, ma meno come poeta lirico. E, in realtà, io sono sempre stato l'una cosa e l'altra, questo, però, non avendo mai il senso di essere diviso in due, di essere lacerato. Per me la prosa, l'esprimermi in prosa, non è in qualche modo diverso dall'esprimermi in poesia, anche se, intendiamoci bene, so perfettamente che una differenza profonda c'è» (G. Bassani, «In rima e senza». Una lezione inedita di Giorgio Bassani dal Fondo di poesia Pietro Tordi, a cura di R. Mosena, «Nuovi Argomenti», cit., p. 77); «In fondo io 
non cambio mai penna. Non ho una penna per la prosa, una penna per la poesia, una penna per i saggi critici, una penna per le lettere. Sono sempre io» (S. Cro, Intervista a Giorgio Bassani, in Lezioni americane di Giorgio Bassani, cit., p. 126); «Non avrei mai potuto scrivere niente se non avessi, prima, scritto Te lucis ante. In un certo senso, è dunque questo il mio libro più importante» (L'Europa letteraria, febbraio 1964; citato in G. Varanini, Giorgio Bassani, Firenze, La Nuova Italia, 1975, p. 8); «Riaffermo che senza aver scritto Te lucis ante non avrei mai potuto scrivere Il romanzo di Ferrara, ad esso successivo. Dentro Te lucis ante c'è, in nuce, tutta la problematica morale, religiosa, che sta alla base del romanzo che ho steso, bada bene, con lo stesso spirito con cui $\mathrm{i}$ poeti di una volta stendevano i loro poemi» (C. F. Colucci, La parola perduta, Napoli, Alfredo Guida editore, 2001, p. 58).

26. G. Bassani, Rolls Royce and Other Poems, Toronto, Aya Press, 1982. Contiene le seguenti poesie tratte da Epitaffio e da In gran segreto: Rolls Royce, Al telefono, Le leggi razziali, Mi chiedi perché mai e quando, L'ho già detto, Davvero cari non saprei dirvelo, Piazza Indipendenza, Saturnia, Campus, A un critico, In gran segreto, Per lettera.

27. Ivi, p. 9.

28. S. Cro, Lettera dell'Editore, «The Canadian Journal of Italian Studies», vol. I, 1977, p. 3. Questo primo numero della rivista canadese è intitolato Omaggio a Giorgio Bassani e contiene i seguenti interventi: G. De Stefanis Oddo, Evoluzione e involuzione nella parabola narrativa di Bassani; S. Gilardino, Giorgio Bassani: una valutazione critica; le quattro poesie ancora inedite, prima di comparire tra quelle di In gran segreto l'anno successivo, sono riunite sotto uno stesso titolo, In Maremma, e comprendono Racconto, La capanna dell'ortolano, In Maremma, Parla il depresso; Intervista con Giorgio Bassani, a cura di S. Cro (ora ripubblicata in Lezioni americane di Giorgio Bassani, cit., pp. 125-134); un altro contributo di Stelio Cro, Tempo e parola nelle «Storie ferraresi», H. Haller, Da «Le storie ferraresi» al «Romanzo di Ferrara»: varianti nell'opera di Bassani.

29. Dalla nota che introduce gli inediti, «The Canadian Journal of Italian Studies», vol. I, $\mathrm{n}^{\circ} 2$, 1978, p. 161. Queste le poesie pubblicate: Compleanno, Campus, Visitando l'Indiana, Per lettera, A un giovane giornalista indiscreto, Da Machado, Shattuck Hotel, In gran segreto. Questi gli articoli contenuti nel numero: M. Schneider, A Conversion to Death: Giorgio Bassani's «L'Airone»; D. Radcliff-Umstead, Transformation in Bassani's Garden; M. Shapiro, Bassani's Ironic Mode; S. Cro, Art and Death in Bassani's Poetry.

30. M. Musa, Poems by Giorgio Bassani, Translated by Mark Musa, «Italian Culture», vol. V, $\mathrm{n}^{\circ} 1,1984$, pp. 109-111. Le poesie tradotte sono: In the Golden Age, Ever Since, I Sweep By Fast As Wind, Believe Me, My Friends, I Can't Begin to Tell You, Italian Forum 1972.

31. G. Bassani, Epitaffio, Milano, Mondadori, 1974, p. 70.

32. Pubblicata nella traduzione di Francesca Valente in G. Bassani, Rolls Royce and Other Poems, cit., p. 27.

33. M. Musa, Poems by Giorgio Bassani, Translated by Mark Musa, cit., p. 111.

34. Questi i suoi libri di poesia: The Mud Actor (Holt, Rinehart and Winston, 1982); Soul Make a Path Through Shouting (Copper Canyon Press, 1994); Beautiful Signor (Copper Canyon Press, 1997); More Than Peace and Cypresses (Copper Canyon Press, 2004); The Crossed-Out Swastika (Copper Canyon Press, 2012); è in uscita The Gospel according to Wild Indigo (Southern Illinois University Press, 2018).

35. Che qui riproduciamo accostandole un nostro tentativo di traduzione.

36. Cyrus Cassells, More Than Peace and Cypresses, Port Townsend, Copper Canyon Press, 2004, pp. 37-38.

37. L. Dalla, Il parco della luna, dall'album Dalla, RCA Italiana, 1980. 


\section{RIASSUNTI}

La poesia e la narrativa di Giorgio Bassani trovano lettori attenti e interessati anche in Canada e negli Stati Uniti soprattutto del sud, dove la denuncia del fascismo e della persecuzione sugli esseri umani viene letta in relazione alla storia degli afro-americani. Come in Bassani, così anche nei writers del sud, troviamo l'impegno costante dell'essere testimoni, di incarnare la memoria, di riaffermare la storia del proprio tempo attraverso l'atto della scrittura. Poesie di Giorgio Bassani sono state tradotte e pubblicate in Nord America negli anni Cinquanta e negli anni Ottanta. In Canada escono, prima ancora che in Italia, alcuni componimenti di In gran segreto e, nel 1982, il libro Rolls Royce and Other Poems. Dall'altra parte dell'Oceano, Bassani ha lasciato un'impronta con le sue visite e le sue esperienze di insegnamento, tracce che mantengono viva la memoria dello scrittore ferrarese.

Bassani's poetry and fiction has found many attentive readers in Canada and in the United States, especially in its Southern states, where Bassani's denunciation of fascism and of persecutions may be connected in some ways with the history of Afro-Americans. In the southern authors, just as in Bassani, the writer is constantly engaged as a witness, who embodies memory itself, and revisits his own time through the very act of writing. Some of Giorgio Bassani's poems were translated and published in the United States first in the fifties and then in the eighties. While in Canada some poems belonging to In Gran Segreto appeared even before than they did in Italy, and the book, Rolls Royce and Other Poems, was published in 1982. On the other side of the Atlantic, Bassani left important marks with his teaching experiences, marks that still today keep his memory alive.

La poésie et les romans de Giorgio Bassani ont trouvé de nombreux lecteurs attentifs et intéressés au Canada et aux États-Unis, surtout dans le sud des États-Unis, où on a pu comparer les persécutions qu'il dénonce à celles subies par les Afro-Américains dans leur pays. Cette dénonciation trouve un écho dans les témoignages des écrivains du Sud qui incarnent la mémoire elle-même et qui réaffirment leur temps à travers l'acte de l'écriture. Certaines poésies de Bassani ont été traduites et publiées aux États-Unis dans les années cinquante et puis dans les années quatre-vingt, alors que certaines des compositions appartenant à In gran segreto voient le jour au Canada avant même de paraître en Italie. Enfin, en 1982, est publié, toujours au Canada, le livre Rolls Royce and Other Poems. Bassani a laissé de l'autre côté de l'océan une trace visible grâce à ses visites et à son expérience d'enseignement, une trace qui maintient sa mémoire encore vivante aujourd'hui.

\section{INDICE}

Mots-clés : Giorgio Bassani, Epitaffio, In gran segreto, poésies, récit de voyage, littérature américaine, Mario Soldati, Emilio Cecchi, traduction

Keywords : Giorgio Bassani, Epitaffio, In gran segreto, poems, travel literature, American literature, Mario Soldati, Emilio Cecchi, translation

Parole chiave : Giorgio Bassani, Epitaffio, In gran segreto, poesie, letteratura di viaggio, letteratura Americana, Mario Soldati, Emilio Cecchi, traduzioni 
AUTORE

VALERIO CAPPOZZO

The University of Mississippi 